Journal for ImmunoTherapy of Cancer

\section{Tumor mutational burden predicts the efficacy of pembrolizumab monotherapy: a pan-tumor retrospective analysis of participants with advanced solid tumors}

To cite: Cristescu R, AuroraGarg D, Albright A, et al. Tumor mutational burden predicts the efficacy of pembrolizumab monotherapy: a pan-tumor retrospective analysis of participants with advanced solid tumors. Journal for ImmunoTherapy of Cancer 2022;10:e003091. doi:10.1136/ jitc-2021-003091

- Additional supplemental material is published online only. To view, please visit the journal online (http://dx.doi.org/10. 1136/jitc-2021-003091).

Accepted 21 December 2021

\section{Check for updates}

(C) Author(s) (or their employer(s)) 2022. Re-use permitted under CC BY-NC. No commercial re-use. See rights and permissions. Published by BMJ.

${ }^{1}$ Merck \& Co, Inc, Kenilworth, New Jersey, USA

${ }^{2}$ MSD China, Beijing, China

Correspondence to Dr Jared Lunceford; jared_lunceford@merck.com

\section{ABSTRACT}

Background Several studies have evaluated the relationship between tumor mutational burden (TMB) and outcomes of immune checkpoint inhibitors. In the phase II KEYNOTE-158 study of pembrolizumab monotherapy for previously treated recurrent or metastatic cancer, high TMB as assessed by the FoundationOne CDx was associated with an improved objective response rate (ORR).

Methods We retrospectively assessed the relationship between TMB and efficacy in participants with previously treated advanced solid tumors enrolled in 12 trials that evaluated pembrolizumab monotherapy, including 3 randomized trials that compared pembrolizumab with chemotherapy. TMB was assessed in formalin-fixed, paraffin-embedded pretreatment tumor samples by whole-exome sequencing. High TMB was defined as $\geq 175$ mutations/exome. Microsatellite instability (MSI) phenotype was based on whole-exome sequencing results. Programmed death ligand 1 (PD-L1) expression was assessed by immunohistochemistry. The primary end point was ORR assessed per RECIST V.1.1 by independent central review. Other end points included progression-free survival (PFS) assessed per RECIST V.1.1 by independent central review and overall survival (OS).

Results Of the 2234 participants in the analysis, 1772 received pembrolizumab monotherapy and 462 received chemotherapy. Among the pembrolizumab-treated participants, ORR was $31.4 \%(95 \% \mathrm{Cl} 27.1$ to 36.0$)$ in the 433 participants with TMB $\geq 175$ mutations/exome and $9.5 \%$ (95\% Cl 8.0 to 11.2$)$ in the 1339 participants with TMB $<175$ mutations/exome. The association of TMB with ORR was observed regardless of PD-L1 expression and not driven by specific tumor types or participants with very high TMB or high MSI. In the 3 randomized controlled trials, TMB was associated with ORR $(p \leq 0.016)$, PFS $(p \leq 0.005)$, and OS $(p \leq 0.029)$ of pembrolizumab but not of chemotherapy $(p \geq 0.340, p \geq 0.643$, and $p \geq 0.174$, respectively), and pembrolizumab improved efficacy versus chemotherapy in participants with TMB $\geq 175$ mutations/ exome.
Conclusions $T M B \geq 175$ mutations/exome is associated with clinically meaningful improvement in the efficacy of pembrolizumab monotherapy and improved outcomes for pembrolizumab versus chemotherapy across a wide range of previously treated advanced solid tumor types. These data suggest TMB has broad clinical utility irrespective of tumor type, PD-L1 expression, or MSI status and support its use as a predictive biomarker for pembrolizumab monotherapy in participants with previously treated advanced solid tumors.

\section{BACKGROUND}

The accumulation of neoantigens, defined as abnormal peptides arising from mutations in genes that encode proteins presented by the major histocompatibility complex, increases the likelihood that tumor cells will be recognized by infiltrating immune cells. Somatic tumor mutational burden (TMB) may result in neoantigen formation with the potential for immune system recognition. 'Therefore, agents that promote T-cell activation such as monoclonal antibodies against programmed death 1 (PD-1) or its ligand, PD-L1, may be particularly effective anticancer therapies in patients with high TMB. Several studies have shown positive correlations between TMB levels and response to immune checkpoint inhibitors across diverse tumor types. ${ }^{2-4}$

The anti-PD-1 monoclonal antibody pembrolizumab received accelerated approval from the US Food and Drug Administration (US FDA) for the treatment of adult and pediatric patients with unresectable or metastatic solid tumors with tumor mutational burdenhigh (TMB-H) ( $\geq 10$ mutations/megabase as determined by an FDA-approved test) that have progressed following prior treatment and who have no satisfactory alternative 
treatment options. The FoundationOne CDx (Foundation Medicine, Cambridge, Massachusetts, USA), a targeted cancer gene panel, was contemporaneously approved as the TMB companion diagnostic for pembrolizumab. Approval was primarily based on a prespecified analysis of patients with select previously treated advanced solid tumors treated with pembrolizumab monotherapy in the phase II KEYNOTE-158 study. ${ }^{5}$ The objective response rate (ORR) was $29 \%$ in the 102 participants with TMB-H tumors per FoundationOne $\mathrm{CDx}$ and $6 \%$ in the 688 participants with non-TMB-H tumors. TMB was predictive of pembrolizumab outcomes irrespective of microsatellite instability (MSI) status, tumor PD-L1 expression, and tumor type.

To explore the value of TMB for predicting response to pembrolizumab monotherapy in cancer types not included in KEYNOTE-158, we assessed the relationship between TMB measured by whole-exome sequencing (WES) and efficacy using individual patient data from 12 additional trials of pembrolizumab monotherapy. ${ }^{6-21}$ Results of this supportive analysis, which used a WES cutpoint that maximized agreement to the 10-mutations/ megabase FoundationOne CDx cutpoint and were submitted to and reviewed by the FDA as part of the application seeking regulatory approval of pembrolizumab for previously treated TMB-H advanced solid tumors, are presented herein.

\section{METHODS}

\section{Trial design, participants, and treatment}

Key eligibility criteria and full protocols for the trials included in this retrospective analysis of participants with previously treated advanced solid tumors have been published. ${ }^{6-21}$ Pembrolizumab was administered intravenously at a dose of $2 \mathrm{mg} / \mathrm{kg}, 10 \mathrm{mg} / \mathrm{kg}$, or $200 \mathrm{mg}$ once every 3 weeks or $10 \mathrm{mg} / \mathrm{kg}$ once every 2 weeks (online supplemental table S1). The chemotherapy regimens in the randomized trials varied by tumor type. ${ }^{91518}$ Where permitted by local laws and regulations, broad genetic/ genomic testing, including WES, was included as a mandatory element of 10 of 12 trials included in this analysis; in KEYNOTE- $002^{8}$ and KEYNOTE- $010,{ }^{9}$ genetic/genomic testing was optional. Only samples provided by properly consented participants were sent for testing.

\section{Genomic profiling}

WES was performed on formalin-fixed, paraffin-embedded pretreatment tumor samples using ImmunoSELECT-RUO (Personal Genome Diagnostics, Baltimore, Maryland, USA) or ACE Cancer Exome (Personalis, Menlo Park, California, USA). After pathology assessment and using a fresh scalpel, the tissue was scraped from the entire section and transferred to a $1.5 \mathrm{~mL}$ tube containing $200 \mu \mathrm{L}$ of $100 \%$ ethanol; if the section contained $<20 \%$ tumor, the tissue was macrodissected from the marked tumor area. DNA was isolated using the QIAamp DNA FFPE Tissue Kit (Qiagen, Valencia, California, USA). Tumor DNA was quantitated using the
Qubit assay (Invitrogen, Carlsbad, California, USA); quality was assessed using the QuantideX qPCR DNA QC Assay (Assuragen, Austin, Texas, USA). WES was performed on matched normal DNA from whole blood collected in a PAXgene DNA Tube (Qiagen) at clinical sites and stored at $-20^{\circ} \mathrm{C}$ or $-70^{\circ} \mathrm{C} / 80^{\circ} \mathrm{C}$ until processed in an approved central laboratory identified by the sponsor. The Chemagic STAR DNA Blood Kit (PerkinElmer, Waltham, Massachusetts, USA) run on either a Hamilton Chemagic STAR or PerkinElmer Chemagic 360 automated instrument was used to extract DNA in a final volume of $500 \mu \mathrm{L}$ or $1.0 \mathrm{~mL}$. Extracted DNA was subjected to volume and concentration determination and ultraviolet and visible spectral analysis to assess purity.

The WES bioinformatics pipeline for analyses across the pembrolizumab translational program has been previously described.$^{22}$ WES reads were aligned to the Genome Reference Consortium Human Build 37 using Burrows-Wheeler Aligner MEM (V.0.7.12) followed by preprocessing steps including duplicate marking, indel realignment, and base recalibration with Picard (V.1.114) and Genome Analysis Toolkit (V.2) to generate analysis-ready binary alignment map (BAM) files. For the $89 \%$ of tumor samples for which matched normal DNA was available, MuTect (V.1.1.7) was used to generate somatic single nucleotide variant (SNV) calls using default parameters by comparing BAM files from tumor and matched normal samples. For the $11 \%$ of tumor samples without matched normal DNA, MuTect (V.1.1.7) was used to call SNVs, which were then analyzed by PureCN (V.1.6.3) to classify germline and somatic status; WES data from 50 platform-matched normal samples were used to build a pool of normal samples to do sequencing error and alignment artifact filtering and copy number normalization. MuTect-called SNVs present in the Single Nucleotide Polymorphism database (V.141) but not in the Catalogue of Somatic Mutations in Cancer (V.68) were filtered out. SNVs with mutant reads $<4$ in tumor samples were also eliminated. The mean coverage was 147 reads per base for tumor and 107 reads per base for normal samples; median coverage was 134 and 105 reads per base, respectively. Overall, 2199 of 2234 tumor samples (99.4\%) had coverage of $>20$ reads per base. Somatic mutations were annotated with Variant Effect Predictor (V.78), and non-synonymous mutations in protein coding regions were counted for TMB. TMB for individual participants was defined as the number of somatic non-synonymous SNVs and indels that passed all described filters. TMB of participants with tumor DNA only was highly concordant with TMB of participants with both tumor and matched normal DNA for a set of 505 tumors analyzed with both pipelines (Spearman's correlation 0.96, with no substantial offset in intercept or slope). There was little association between computational tumor purity and TMB (Spearman's correlation 0.22).

The FoundationOne CDx was performed at the Clinical Laboratory Improvement Amendments-certified laboratory of Foundation Medicine using the Dx1 baitset. TMB was calculated by counting the number of synonymous and non-synonymous mutations across an $0.8 \mathrm{Mb}$ 
region spanning 324 genes, with computational germline status and oncogenic driver filtering.

Participants were evaluable for TMB if their samples provided valid TMB scores on sequencing and analysis. Participants were non-evaluable for TMB if they did not provide any tumor tissue, if they did not consent to exploratory biomarker research, if the amount of tumor tissue was inadequate for analysis, if the samples did not yield sufficient DNA from either tumor tissue or matched normal DNA for WES, or if the samples failed sequencing and subsequent quality control steps.

Expression of an 18-gene T-cell inflamed gene expression profile (GEP) was assessed using the NanoString nCounter gene expression platform (NanoString Technologies, Seattle, Washington, USA) as described by Ayers $e t a l^{23}$ in RNA isolated from formalin-fixed, paraffinembedded tumor samples (processed as outlined above for WES). MSI genotype was detected by applying mSINGS on WES data from tumor samples. The stability of each mononucleotide microsatellite locus was evaluated, and the proportion of unstable microsatellite loci was determined as the MSI score. MSI was confirmed by PCR using the Promega MSI Analysis System, V.1.2, in the 304 pembrolizumab-treated participants from KEYNOTE-059 $9^{17}$ and KEYNOTE-061 $1^{18}$ included in the analysis.

\section{Histology}

PD-L1 expression was assessed by immunohistochemistry in formalin-fixed tumor samples using either the PD-L1 IHC 22C3 pharmDx assay (Agilent, Carpinteria, California, USA) or a laboratory-developed prototype assay based on the 22C3 antibody (Qualtek, Goleta, California, USA). The definition of positivity using the PD-L1 IHC 22C3 pharmDx assay for all tumors except melanoma and non-small-cell lung cancer was combined positive score (CPS) $\geq 1$, where CPS was defined as the number of PD-L1-expressing tumor cells, lymphocytes, and macrophages divided by the total number of viable tumor cells, multiplied by 100 . For melanoma, positivity was defined as Allred proportion score $\geq 2$, which is equivalent to membranous PD-L1 expression in $\geq 1 \%$ of tumor and tumor-associated immune cells; for non-small-cell lung cancer, positivity was defined as tumor proportion score (TPS) $\geq 1 \%$, where TPS was defined as the percentage of PD-L1-expressing tumor cells out of the total number of viable tumor cells. The definition of positivity using the prototype assay was membranous PD-L1 expression on $\geq 1 \%$ of tumor and associated inflammatory cells or positive staining in the stroma.

\section{Statistical analysis}

Individual participant data from all pembrolizumab monotherapy trials that included patients with previously treated advanced solid tumors for which WES data were available at the time the TMB regulatory submission package was prepared in 2020 were assembled as supportive evidence and reviewed by the FDA. Data from the KEYNOTE-002 chemotherapy group ${ }^{8}$ were excluded because there were too few participants with available TMB for reliable between-arm treatment comparisons. Data from KEYNOTE-199 cohort $3^{21}$ were excluded because participants had bone-predominant metastatic castration-resistant prostate cancer, precluding them from being assessed for the primary end point of this analysis. The analysis population included participants with available TMB scores who received $\geq 1$ dose of study treatment and received prior treatment for their cancer.

Among a range of cutoffs showing clear enrichment for objective response, the WES TMB cutpoint was selected in a training set of participants treated with pembrolizumab monotherapy by evaluating the relationship between TMB and inflammation in the tumor microenvironment as assessed using an 18-gene T-cell inflamed GEP $^{23}$ using a method similar to that of Panda et al. ${ }^{24}$ The WES cutpoint that maximized agreement to the 10-mutations/megabase FoundationOne CDx cutpoint was assessed in participants in the training set who had TMB assessed by both methods. ${ }^{525}$

Statistical analyses were performed using SAS V.9.4 (SAS Institute, Cary, North Carolina, USA). The primary end point was ORR (ie, proportion of participants with confirmed complete or partial response) assessed per RECIST V.1.1 by independent central review. Other end points were duration of response (DOR, ie, time from initial response to disease progression or death in participants with complete or partial response) and progressionfree survival (PFS, ie, time from randomization (first dose of pembrolizumab in non-randomized trials) to disease progression or death) assessed per RECIST V.1.1 by independent central review and overall survival (OS, ie, time from randomization (first dose of pembrolizumab in nonrandomized trials) to death). The Kaplan-Meier method was used to estimate DOR, PFS, and OS. ORR, DOR, PFS, and OS were summarized descriptively for participants with $\mathrm{TMB} \geq 175$ and $<175$ mutations/exome. For analyses by tumor type, tumor types represented by $\leq 10$ participants were pooled into a single category termed 'other'. Although our focus was to provide clinical findings for the 175 mutations/exome WES TMB cutpoint that was aligned with the 10-mutations/megabase FoundationOne CDx cutpoint, we have also provided a profile of ORR in patients with TMB above a range of progressively higher cutoffs, beginning with 0 and increasing in increments of 25 mutations/ exome, for the pooled dataset along with pointwise $95 \%$ CIs; at least 50 participants must have had TMB above the cut-off for estimating ORR and the CI. The impact of key baseline characteristics on the robustness of the relationship between TMB status and response to pembrolizumab was assessed using logistic regression analysis that included the following covariates: TMB status $(\geq 175 \mathrm{vs}<175$ mutations/exome), PD-L1 expression status (positive vs negative), MSI status (high vs non-high), age ( $<65$ vs $\geq 65$ years), sex (female vs male), Eastern Cooperative Oncology Group (ECOG) performance status ( 0 vs 1 or 2 ), number of lines of prior therapy for advanced disease ( $\leq 1 \mathrm{vs} \geq 2$ ), and tumor 
type (model parameterized as difference relative to gastric cancer).

The treatment effect of pembrolizumab versus chemotherapy was assessed in the 3randomized studies ${ }^{915} 18$ by comparing ORR, DOR, PFS, and OS in participants with TMB $\geq 175$ and $<175$ mutations/exome. To evaluate associations between TMB and efficacy by treatment, logistic regression analyses adjusted for ECOG performance status were performed separately in participants treated with pembrolizumab and in participants treated with chemotherapy for each randomized study; two-sided $\mathrm{p}$ values (not adjusted for multiplicity) are presented as a measure of the strength of association between each efficacy end point and TMB assessed as a continuous, $\log _{10^{-}}$ transformed variable.

All genomic and clinical data were provided to and reviewed by the FDA as part of the application seeking regulatory approval of pembrolizumab for previously treated TMB-H advanced solid tumors.

\section{Reanalysis of data from Samstein et al}

Data from Samstein et $a l^{26}$ were downloaded from http:/ / www.cbioportal.org/study?id=tmb_mskcc_2018 in September 2019. The only publicly available data were for the 1662 participants treated with an immune checkpoint inhibitor as monotherapy or in combination. Cox regression analyses associating high versus low TMB with OS across tumor types, within tumor types, and at various TMB cutpoints were executed in MATLAB (MathWorks, Natick, Massachusetts, USA) and HRs and $95 \%$ CIs were reported. Because data for estrogen receptor status were not provided in the downloaded dataset, we could not perform analyses

A

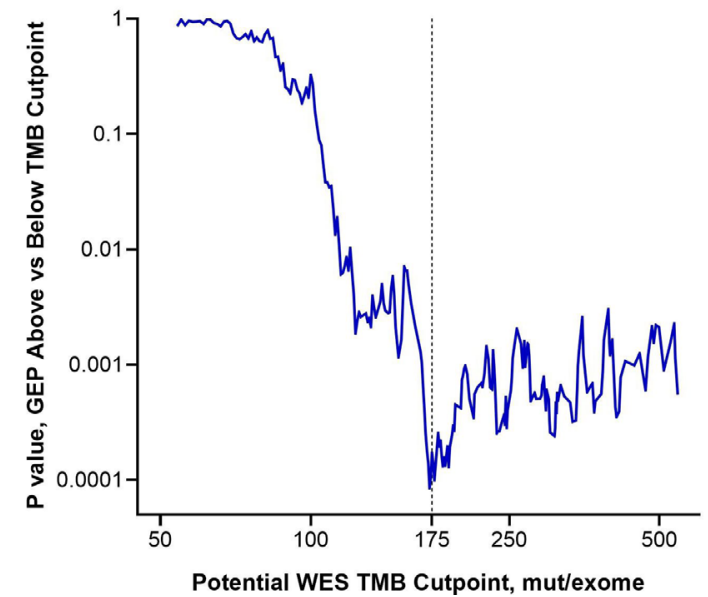

for estrogen receptor-positive and estrogen receptornegative breast cancer. Using the published tumorspecific cutoffs, we observed HRs and 95\% CIs that qualitatively appeared identical to those reported by Samstein et $a l . .^{26}$ After this verification, we analyzed the data using a single, pan-tumor cutpoint of 10 mutations/megabase and plotted the resultant HRs and 95\% CIs against those we obtained using the tumor-specific cutoffs (online supplemental figure S1). ${ }^{26}$

\section{RESULTS}

\section{Cutpoint verification}

We verified WES TMB 175 mutations/exome as the optimal cutpoint in distinct analyses. One analysis used a training set that included the first 976 participants with previously treated cancer for whom TMB was obtained, of whom 810 also had GEP data. We found that WES TMB 175 mutations/exome enriched for response to pembrolizumab and was associated with peak statistical significance for differences in inflammation in the tumor microenvironment as measured by the 18-gene T-cell inflamed GEP (figure 1A). In the second analysis, which included the 338 participants in the training set who had TMB assessed by both WES and FoundationOne CDx, there was high concordance between TMB assessed by both methods (Spearman's correlation, 0.71), and 175 mutations/exome was the WES cutpoint that maximized average positive and negative agreement with the 10-mutations/megabase FoundationOne CDx cutpoint (area under the receiver-operating characteristics curve, 0.92) (figure 1B).

B

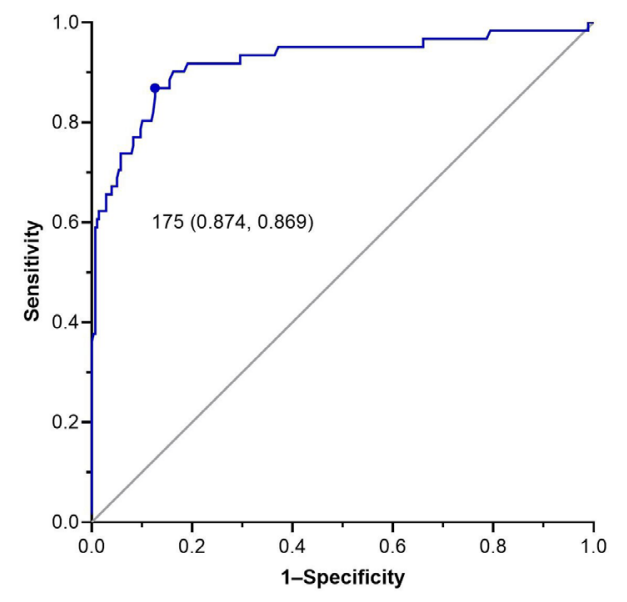

Figure 1 Validation of the optimal cutpoint for TMB assessed by WES. (A) Comparison of inflammation in the tumor microenvironment assessed using an 18-gene T-cell inflamed GEP in participants with TMB above versus below potential WES cutpoints for mutational burden. $P$ values are two-sided and assessed using the Wilcoxon rank-sum test. The vertical dotted line represents TMB 175 mut/exome. (B) The optimal TMB cut-off assessed by WES that corresponds with the FoundationOne CDx TMB cutpoint of 10 mut/megabase. The Youden index of 175 mut/exome assessed per WES is the point of maximal average positive and negative agreement rates with 10 mut/megabase by the FoundationOne CDx. The area under the receiveroperating characteristic curve is 0.92 . GEP, gene expression profile; mut, mutations; TMB, tumor mutational burden; WES, whole-exome sequencing. 


\section{Clinical utility of the cutpoint}

To assess the clinical utility of the 175-mutations/ exome cutpoint, we explored efficacy in a pooled population of participants with previously treated cancer enrolled in 12 clinical trials-9 single-arm trials of pembrolizumab monotherapy and 3 randomized trials that compared pembrolizumab monotherapy with chemotherapy and enrolled participants between 2011 and 2017 (online supplemental table S1). Including the 876 eligible participants from the training set, 2234 participants representing 24 tumor types-1772 who received pembrolizumab monotherapy and 462 who received chemotherapy-were included in the WES TMB analysis population (online supplemental figure S2). Median (IQR) follow-up duration, defined as time from the first dose of study treatment to data cut-off, was 26.1 months (20.1-32.9). Among 1772 pembrolizumab-treated participants, $433(24.4 \%)$ had TMB $\geq 175$ mutations/exome and $1339(75.6 \%)$ had TMB $<175$ mutations/exome (online supplemental table S2). Among 462 chemotherapy-treated participants, 140 (30.3\%) had TMB $\geq 175$ mutations/exome and $322(69.7 \%)$ had TMB $<175$ mutations/exome (online supplemental table S2). The prevalence of TMB $\geq 175$ mutations/exome among tumor types represented by $>10$ participants ranged from $4.1 \%$ (ovarian cancer) to $56.6 \%$ (melanoma) (online supplemental table S2). The most prevalent tumor types in the TMB $\geq 175$ and $<175$ mutations/exome populations were non-small-cell lung cancer and ovarian cancer, respectively (figure 2). Aside from imbalances in sex, baseline characteristics were generally similar in the TMB $\geq 175$ and $<175$ mutations/exome populations (online supplemental table S3). Twenty-one (1.2\%) participants, all of whom had TMB $\geq 175$ mutations/ exome, had MSI-high (MSI-H) tumors per WES. More participants in the $\geq 175 \mathrm{vs}<175$ mutations/exome population completed $(7.4 \%$ vs $1.6 \%)$ or remained on $(8.5 \%$ vs $4.1 \%)$ pembrolizumab at the time of data cutoff (online supplemental table S4).

In the pooled pembrolizumab population, we found that participants with TMB $\geq 175$ mutations/exome had better outcomes than those with TMB $<175$ mutations/ exome. ORR was $31.4 \%$ (95\% CI 27.1 to 36.0 ) in participants with $\mathrm{TMB} \geq 175$ mutations/exome and $9.5 \%(95 \%$ CI 8.0 to 11.2 ) in those with TMB $<175$ mutations/exome (online supplemental table S5). Reductions from baseline in target lesion size were observed in $67.5 \%$ and $41.5 \%$ of participants, respectively (online supplemental figure S3). Excluding participants from the training set, ORR was $26.3 \%$ (95\% CI 20.3 to 33.0 ) and $10.2 \%$ (95\% CI 8.0 to 12.7$)$ in the $\geq 175$ mutations/exome ( $n=198)$ and $<175$ mutations/exome ( $\mathrm{n}=698$ ) populations, respectively. In the pooled population of 1772 participants, ORR increased as the WES TMB cutpoint was increased (online supplemental figure S4).

We confirmed that the response enrichment provided by TMB $\geq 175$ mutations/exome was robust and not driven by associations with other confounding variables using subgroup analyses, participant-level data, and logistic regression. TMB $\geq 175$ mutations/exome enriched for response in each of the 8 tumor types that included $>10$ participants and in the pooled set of 16 tumor types that included $\leq 10$ participants each (figure 3A). Participantlevel data showed that the overall association of TMB with ORR was not driven by outliers with very high TMB or by specific tumor types (figure 3B). The ORR was higher among participants with TMB $\geq 175$ mutations/exome regardless of PD-L1 status (figure 3C) or MSI genotype (figure 3D). In a multivariate logistic regression analysis, the OR for ORR in participants with TMB $\geq 175$ vs $<175$ mutations/exome was 3.076 (95\% CI 2.243 to 4.220 )
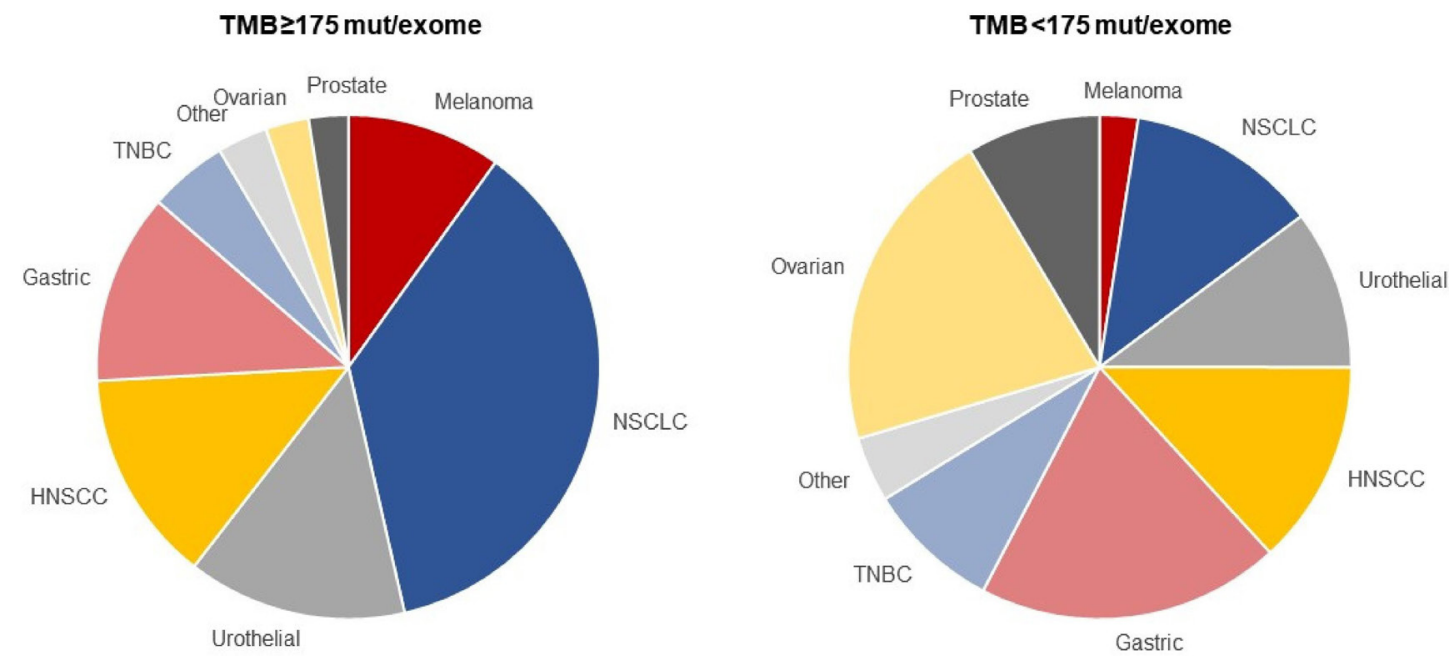

Figure 2 Proportion of participants in the pooled pembrolizumab population with whole-exome sequencing TMB $\geq 175$ mut/exome and TMB $<175$ mut/exome by tumor type. The tumor types are presented in order of descending median tumor mutational burden. For a listing of tumor types included in the 'other' category, see online supplemental table S2. HNSCC, head and neck squamous cell carcinoma; mut, mutations; NSCLC, non-small-cell lung cancer; TMB, tumor mutational burden; TNBC, triple-negative breast cancer. 
A

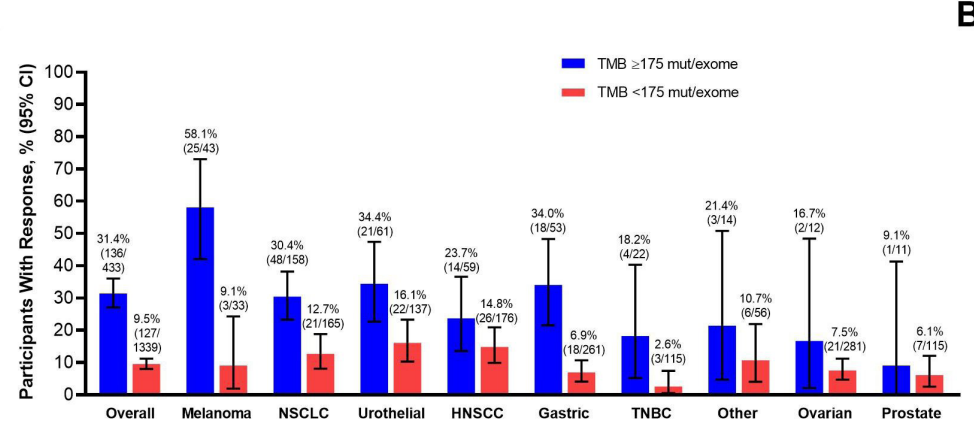

B

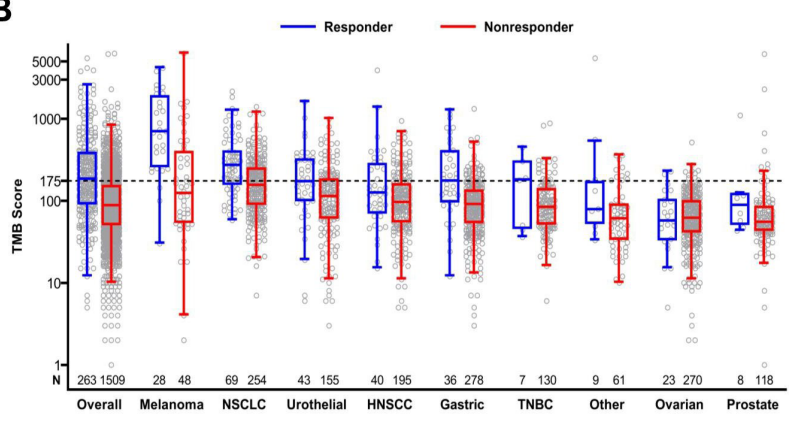

C

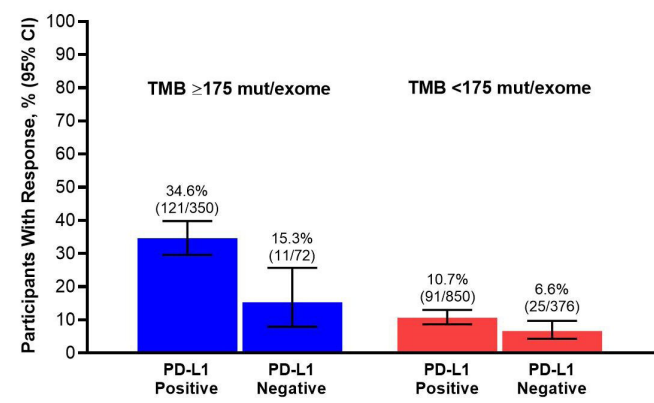

D

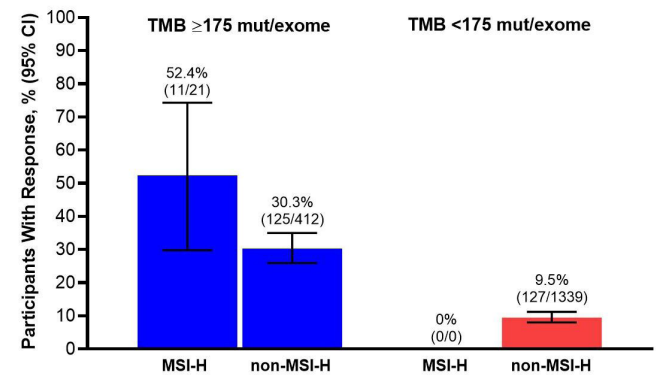

Figure 3 Objective response rate in participants in the pooled pembrolizumab population by TMB $\geq 175$ mut/exome and $<175$ mut/exome. (A) ORR in the overall population and by tumor type. Tumor types are presented in order of descending median TMB; for a listing of tumor types included in the 'other' category, see online supplemental table S2. (B) Participant-level TMB scores in responders and non-responders overall and by tumor type. Tumor types are presented in order of descending median TMB; for a listing of tumor types included in the 'other' category, see online supplemental table S2. The center line represents the median, the box limits represent the upper and lower quartiles, and the whiskers represent the maximum within $1.5 \times$ IQR from the 75th percentile and the minimum within 1.5xIQR from the 25th percentile. (C) ORR in the overall population by PDL1 expression status. (D) ORR in the overall population by MSI genotype. HNSCC, head and neck squamous cell carcinoma; MSI-H, microsatellite instability high; mut, mutations; NSCLC, non-small-cell lung cancer; ORR, objective response rate; PD-L1, programmed death ligand 1; TMB, tumor mutational burden; TNBC, triple-negative breast cancer.

after adjusting for the effects of other prognostic variables (table 1).

Among responders, median DOR was not reached in the TMB $\geq 175$ mutations/exome population and was 12.5 months in the TMB $<175$ mutations/exome population; $63.8 \%$ and $32.2 \%$ of responses, respectively, were estimated to last for $\geq 24$ months (figure $4 \mathrm{~A}$ ). Median PFS was 4.2 months (95\% CI 3.6 to 5.8) in participants with TMB $\geq 175$ mutations/exome and 2.1 months (95\% CI 2.0 to 2.1) in participants with TMB $<175$ mutations/exome (figure 4B). Median OS was 15.5 months (95\% CI 13.1 to 17.7 ) and 10.1 months (95\% CI 9.4 to 10.9), respectively; the estimated percentage of participants alive at 24 months was $40.0 \%$ and $23.2 \%$ (figure 4C).

To further assess the association between TMB and outcomes, we used exploratory regression models and data from the 3randomized clinical trials, which enrolled patients with non-small-cell lung cancer (KEYNOTE-010), ${ }^{9}$ urothelial cancer (KEYNOTE-045), ${ }^{15}$ and gastric cancer (KEYNOTE-061). ${ }^{18}$ In these trials, TMB as a continuous $\log _{10}$-transformed variable was significantly associated with ORR $(\mathrm{p} \leq 0.016)$, PFS $(\mathrm{p} \leq 0.005)$, and OS $(\mathrm{p} \leq 0.029)$ in the pembrolizumab groups but not the chemotherapy groups ( $\mathrm{p} \geq 0.340,0.643$, and 0.174 , respectively) (online supplemental table $\mathrm{S} 6$ ). The benefit of pembrolizumab versus chemotherapy was enhanced in participants with TMB $\geq 175$ mutations/exome in all 3randomized trials (table 2; figure 5, online supplemental figure S5, S6).

\section{DISCUSSION}

In this retrospective analysis of individual participant data from 12 clinical trials across 24 tumor types that contributed to the US FDA approval of pembrolizumab monotherapy for the treatment of patients with unresectable or metastatic TMB-H solid tumors that progressed following prior treatment and who have no satisfactory treatment options, we found that TMB $\geq 175$ mutations/exome assessed by WES was predictive of outcomes to pembrolizumab. TMB $\geq 175$ mutations/exome enriched for response to pembrolizumab across tumor types, including those with generally high TMB like melanoma and those with generally low TMB like ovarian cancer. ${ }^{27}{ }^{28} \mathrm{TMB} \geq 175$ mutations/ exome was also associated with longer DOR, PFS, and OS, although pooled OS estimates should be interpreted with caution given the different representation of tumor 
Table 1 Multivariate logistic regression analysis of response to pembrolizumab assessed per RECIST V.1.1 by independent central review in the pooled pembrolizumab population

\begin{tabular}{|c|c|}
\hline Covariate & OR for ORR $(95 \% \mathrm{Cl})$ \\
\hline $\begin{array}{l}\text { TMB status ( } \geq 175 \text { vs } \\
<175 \text { mutations/exome) }\end{array}$ & 3.076 (2.243 to 4.220$)$ \\
\hline $\begin{array}{l}\text { PD-L1 expression status } \\
\text { (positive vs negative) }\end{array}$ & 2.187 (1.442 to 3.316$)$ \\
\hline MSI status (high vs non-high) & 3.798 (1.453 to 9.925$)$ \\
\hline Age (<65 vs $\geq 65$ years) & 0.707 (0.525 to 0.952$)$ \\
\hline Sex (female vs male) & $1.004(0.713$ to 1.413$)$ \\
\hline $\begin{array}{l}\text { ECOG performance status } \\
(0 \text { vs } 1 \text { or } 2)\end{array}$ & 1.471 (1.094 to 1.978$)$ \\
\hline $\begin{array}{l}\text { No. of lines of prior therapy for } \\
\text { advanced disease }(\leq 1 \text { vs } \geq 2)\end{array}$ & 1.237 (0.898 to 1.705$)$ \\
\hline Tumor type (HNSCC vs gastric*) & $1.841(1.057$ to 3.207$)$ \\
\hline Tumor type (melanoma vs gastric ${ }^{\star}$ ) & 3.664 (1.877 to 7.153$)$ \\
\hline Tumor type (NSCLC vs gastric*) & $1.617(0.971$ to 2.695$)$ \\
\hline Tumor type (other vs gastric ${ }^{\star}$ ) & 1.203 (0.517 to 2.802$)$ \\
\hline Tumor type (ovarian vs gastric ${ }^{\star}$ ) & $0.882(0.415$ to 1.878$)$ \\
\hline Tumor type (prostate vs gastric ${ }^{\star}$ ) & $0.718(0.300$ to 1.719$)$ \\
\hline Tumor type (TNBC vs gastric*) & $0.526(0.211$ to 1.312$)$ \\
\hline Tumor type (urothelial vs gastric*) & 2.224 (1.291 to 3.832$)$ \\
\hline
\end{tabular}

*Gastric cancer was chosen as the reference because alphabetically, it was the first tumor type group included in the analysis.

ECOG, Eastern Cooperative Oncology Group; HNSCC, head and neck squamous cell carcinoma; MSI, microsatellite instability; NSCLC, non-small-cell lung cancer; ORR, objective response rate; $\mathrm{PD}-\mathrm{L} 1$, programmed death ligand $1 ; \mathrm{TMB}$, tumor mutational burden; TNBC, triple-negative breast cancer.

types with differing prognosis in the TMB $\geq 175$ and $<175$ mutations/exome populations. Our finding that TMB was consistently and significantly associated with efficacy of pembrolizumab but not of chemotherapy and that the relative benefit of pembrolizumab versus chemotherapy was enhanced in participants with $\mathrm{TMB} \geq 175$ mutations/ exome across the 3randomized trials included in our analysis supports the clinical utility of TMB as a predictive biomarker for pembrolizumab monotherapy.

Importantly, the association of $\mathrm{TMB}$ with response in our study was not driven by outliers with very high TMB, by the minority of participants with MSI-H tumors, or by specific tumor types. Although ORR was higher among participants with PD-L1-positive tumors, TMB $\geq 175$ mutations/exome enriched for response regardless of PD-L1 expression. After adjusting for key baseline demographics and disease characteristics, including PD-L1 expression, the adjusted OR for response to pembrolizumab in participants with TMB $\geq 175$ versus $<175$ mutations/exome was 3.076, confirming that the enrichment in response provided by high $\mathrm{TMB}$ is robust and not driven by associations of confounding variables. Together with a growing body of evidence suggesting that TMB and PD-L1 expression are not correlated but are jointly informative for clinical outcomes in patients treated with antiPD-1 or anti-PD-L1 monotherapy ${ }^{29-33}$ and the findings from KEYNOTE-158 showing that high TMB expression is predictive of response to pembrolizumab in a PD-L1unselected population, ${ }^{5}$ our data support the use of TMB as a clinically meaningful biomarker regardless of PD-L1 expression.

We verified that TMB 175 mutations/exome assessed by WES is well aligned with the FoundationOne CDx TMB cutpoint of 10 mutations/megabase that is known to enrich for response, ${ }^{34}$ is the cut-off agreed on by a multistakeholder group convened by the Friends of Cancer Research to harmonize on a standard cut-off for clinical trials, ${ }^{25}$ and is the cutpoint used in the KEYNOTE-158 TMB analysis. ${ }^{5}$ The high concordance we observed between TMB assessed by WES and the FoundationOne CDx suggests that molecular biomarker discoveries made using exploratory techniques such as WES may
A

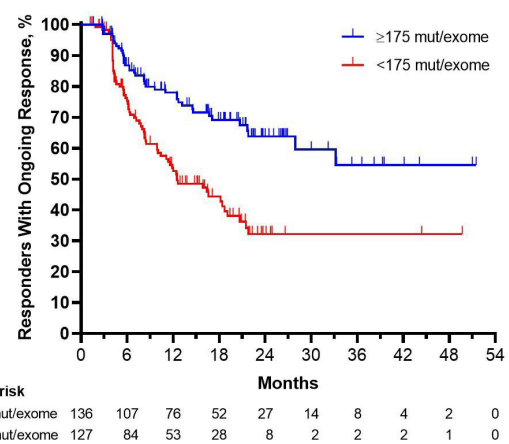

B

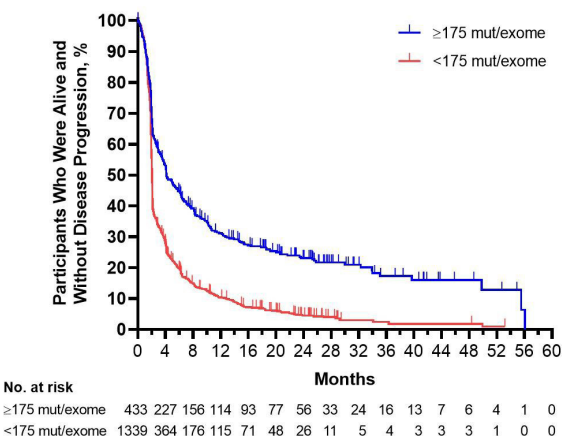

C

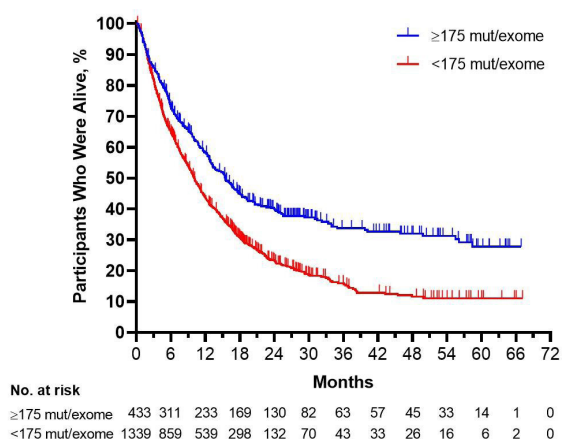

Figure 4 Longitudinal outcomes in participants in the pooled pembrolizumab population by TMB $\geq 175$ mut/exome and $<175$ mut/exome. (A) Kaplan-Meier estimates of duration of response assessed per RECIST V.1.1 by independent central review among participants in the pooled pembrolizumab population with a complete or partial response. (B) Kaplan-Meier estimates of progression-free survival assessed per RECIST V.1.1 by independent central review in the overall pooled pembrolizumab population. (C) Kaplan-Meier estimates of overall survival in the overall pooled pembrolizumab population. mut, mutations; TMB, tumor mutational burden. 
Table 2 Efficacy of pembrolizumab versus chemotherapy in the 3randomized clinical trials included in the analysis by $\mathrm{TMB} \geq 175$ mutations/exome and TMB $<175$ mutations/exome

\begin{tabular}{|c|c|c|c|c|c|c|}
\hline \multirow[b]{2}{*}{ Study } & \multicolumn{3}{|c|}{ TMB $\geq 175$ mutations/exome } & \multicolumn{3}{|c|}{ TMB < 175 mutations/exome } \\
\hline & $\begin{array}{l}\text { ORR, OR } \\
(95 \% \mathrm{Cl})\end{array}$ & $\begin{array}{l}\text { PFS, HR } \\
\text { (95\% Cl) }\end{array}$ & $\begin{array}{l}\text { OS, HR } \\
\text { (95\% Cl) }\end{array}$ & $\begin{array}{l}\text { ORR, OR } \\
(95 \% \mathrm{Cl})\end{array}$ & $\begin{array}{l}\text { PFS, HR } \\
(95 \% \mathrm{Cl})\end{array}$ & $\begin{array}{l}\text { OS, HR } \\
\text { (95\% Cl) }\end{array}$ \\
\hline KEYNOTE-010 & $2.82(0.98$ to 8.11$)$ & 0.59 (0.40 to 0.87$)$ & 0.56 (0.38 to 0.83$)$ & 0.76 (0.29 to 2.00$)$ & 1.09 (0.72 to 1.63$)$ & 0.85 (0.56 to 1.30$)$ \\
\hline KEYNOTE-045 & 3.05 (1.20 to 7.79$)$ & 0.62 (0.40 to 0.96$)$ & $0.63(0.40$ to 1.00$)$ & $1.10(0.55$ to 2.21$)$ & $1.13(0.87$ to 1.47$)$ & 0.71 (0.54 to 0.94$)$ \\
\hline KEYNOTE-061 & $3.43(0.99$ to 11.85$)$ & 0.73 (0.44 to 1.22$)$ & 0.46 (0.27 to 0.81$)$ & 0.61 (0.31 to 1.22$)$ & 1.78 (1.43 to 2.22$)$ & $1.12(0.90$ to 1.41$)$ \\
\hline
\end{tabular}

ORR and PFS were assessed per RECIST V.1.1 by independent central review.

ORR, objective response rate; PFS, progression-free survival; TMB, tumor mutational burden.

be successfully translated to smaller cancer gene panels like FoundationOne CDx, which are more easily implemented in routine clinical practice and may be more costeffective. $^{35} 36$

While other studies also show that a single TMB cutpoint enriches for outcomes of checkpoint inhibitors regardless of tumor type, ${ }^{5} 24$ 37-40 there are reports that suggest tumor-specific cutpoints are needed. ${ }^{26} 40-42$ When we reanalyzed the publicly available data from one such study, published by Samstein $e t a l,{ }^{26}$ we found that a 10-mutations/megabase cutpoint was associated with improved OS across tumor types, with HRs for death generally consistent with those based on the originally reported tumor-specific cutpoints (online supplemental figure S1). The results of our study and our reanalysis of the Samstein et al data show that although the distribution of TMB scores varies across tumor types, a single cutpoint that provides clinically meaningful enrichment of response to PD-1 blockade across tumor types can be identified. This highlights the importance of distinguishing between the conditional distribution of response associated with a biomarker and the marginal distribution of the biomarker itself. Identification of a single cutpoint with broad clinical utility is valuable for fully realizing the

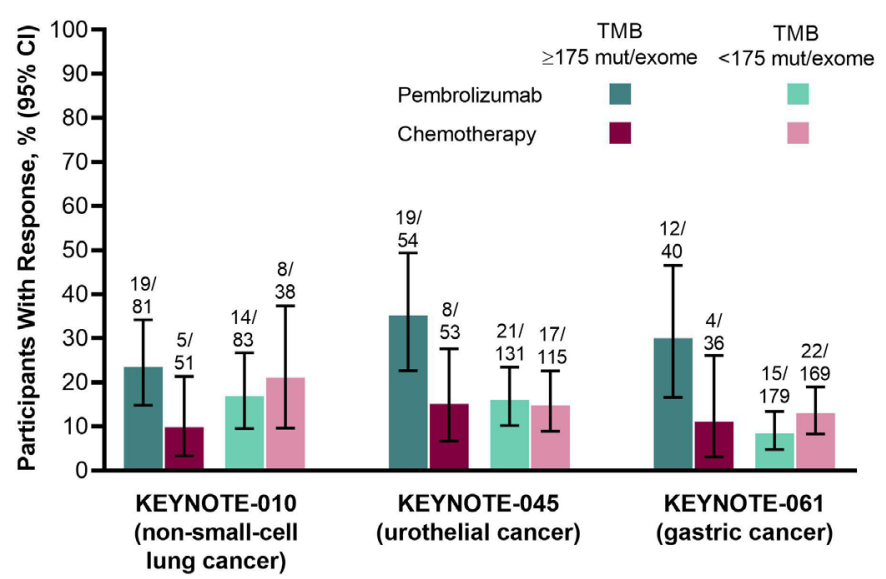

Figure 5 Objective response rate by treatment arm in participants in the 3randomized clinical trials included in the analysis by TMB $\geq 175$ mut/exome and $<175$ mut/exome. Response was assessed per RECIST V.1.1 by independent central review. mut, mutations; NSCLC, non-small-cell lung cancer; TMB, tumor mutational burden. initial diagnostic implementation of TMB as a predictive biomarker.

One limitation of our analysis is the inclusion of 876 participants from the training set in the overall pooled pembrolizumab population $(\mathrm{n}=1772)$. However, the ORR in the TMB $\geq 175$ and $<175$ mutations/exome populations was similar regardless of whether participants from the training set were included in the analysis. Another limitation is the inclusion of tumors without matched normal DNA in our analyses; the low frequency of these samples and the high concordance of TMB derived from the tumor-only and matched-normal pipelines suggests that their inclusion did not bias the analysis. Although the data set includes tumor types for which pembrolizumab is already approved and could be biased by the tumor types for which pembrolizumab trials are available and the participants in the trials for whom WES data were available, we believe that a population of almost 2500 participants that includes 24 tumor types with a mix of those associated with high TMB (eg, melanoma, non-small-cell lung cancer, and urothelial cancer) and those associated with generally low TMB (eg, triple-negative breast, ovarian, and prostate cancers) is robust and supports the ability of TMB to predict response to pembrolizumab regardless of tumor type. While formal statistical testing of the predictive value of TMB was not specified, logistic regression analysis that included key baseline demographics and disease characteristics was performed, and the resultant OR estimates confirm the robustness of our findings.

In conclusion, our data show that a single TMB cutpoint determined by WES that has broad clinical utility irrespective of tumor type, PD-L1 expression, or MSI genotype can be identified, mapped to a targeted cancer gene panel, and used to define a population that derives clinically meaningful benefit from pembrolizumab monotherapy. Our data support TMB as a predictive biomarker for pembrolizumab and the FDA approval of pembrolizumab monotherapy for the treatment of patients with unresectable or metastatic, TMB-H solid tumors that have progressed following prior treatment and who have no satisfactory alternative treatment options.

Acknowledgements The authors would like to thank the patients and their families and caregivers for participating in the clinical trials; the investigators 
and site personnel of each clinical trial; all sponsor personnel who contributed to the clinical trials included in the analysis; and the following current or former employees of Merck Sharp \& Dohme Corp, a subsidiary of Merck \& Co, Inc, Kenilworth, New Jersey, USA: Michael Morrissey, Caroline Fox, and Dean Li for supporting the data collection process; Ronghua Chen and Xinwei Sher for support with the bioinformatics pipeline; Jonathan D Cheng, Scot Ebbinghaus, and Jeffrey Evelhoch for critical review of the manuscript; and Melanie A Leiby for medical writing and editorial assistance.

Contributors RC, DA-G, AA, XQL, AS, and JL acquired the data. RC, DA-G, XQL, AL, FJ, AS, and JL analyzed the data. RC, DA-G, AA, AL, FJ, EHR, AS, and JL interpreted the data. LX performed the statistical analyses. RC, DA-G, AS, and JL drafted the manuscript. All authors contributed to interpreting the results and editing the manuscript for intellectual content and approved the submitted version. $\mathrm{JL}$ is responsible for the overall work as the guarantor.

Funding All clinical studies included in the analysis were funded by Merck Sharp \& Dohme Corp, a subsidiary of Merck \& Co, Inc, Kenilworth, New Jersey, USA. ClinicalTrials.gov numbers for included studies are: NCT01295827, NCT01704287, NCT01905657, NCT01848834, NCT02054806, NCT02256436, NCT02255097, NCT02335411, NCT02370498, NCT02447003, NCT02674061, NCT02787005.

Competing interests RC, DA-G, AA, LX, AL, LL, FJ, EHR, and JL are full-time employees of Merck Sharp \& Dohme Corp, a subsidiary of Merck \& Co, Inc, Kenilworth, New Jersey, USA and hold stock in Merck \& Co, Inc, Kenilworth, New Jersey, USA. XQL is a full-time employee of MSD China. AS was a fulltime employee of Merck Sharp \& Dohme Corp, a subsidiary of Merck \& Co, Inc, Kenilworth, New Jersey, USA at the time the study was conducted.

\section{Patient consent for publication Not applicable.}

Ethics approval All clinical trial participants provided written informed consent before enrollment. All study protocols were consistent with the global standards of the International Conference on Harmonization Good Clinical Practices, the Council for International Organizations of Medical Public Policy Statement: Clinical Trial Ethics Sciences International Ethical Guidelines for Biomedical Research Involving Human Subjects, the Pharmaceutical Research and Manufacturers of America Principles on Conduct of Clinical Trials, applicable local regulatory requirements, and the ethical principles that have their origin in the Declaration of Helsinki.

Provenance and peer review Not commissioned; externally peer reviewed.

Data availability statement Data are available on reasonable request. Merck Sharp \& Dohme Corp, a subsidiary of Merck \& Co, Inc, Kenilworth, New Jersey, USA (MSD) is committed to providing qualified scientific researchers access to anonymized data and clinical study reports from the company's clinical trials for the purpose of conducting legitimate scientific research. MSD is also obligated to protect the rights and privacy of trial participants and, as such, has a procedure in place for evaluating and fulfilling requests for sharing company clinical trial data with qualified external scientific researchers. The MSD data sharing website (available at: http://engagezone.msd.com/ds_documentation.php) outlines the process and requirements for submitting a data request. Applications will be promptly assessed for completeness and policy compliance. Feasible requests will be reviewed by a committee of MSD subject matter experts to assess the scientific validity of the request and the qualifications of the requestors. In line with data privacy legislation, submitters of approved requests must enter into a standard data-sharing agreement with MSD before data access is granted. Data will be made available for request after product approval in the USA and European Union or after product development is discontinued. There are circumstances that may prevent MSD from sharing requested data, including country-specific or region-specific regulations. If the request is declined, it will be communicated to the investigator. Access to genetic or exploratory biomarker data requires a detailed, hypothesis-driven statistical analysis plan that is collaboratively developed by the requestor and MSD subject matter experts; after approval of the statistical analysis plan and execution of a data-sharing agreement, MSD will either perform the proposed analyses and share the results with the requestor or will construct biomarker covariates and add them to a file with clinical data that is uploaded to an analysis portal so that the requestor can perform the proposed analyses.

Supplemental material This content has been supplied by the author(s). It has not been vetted by BMJ Publishing Group Limited (BMJ) and may not have been peer-reviewed. Any opinions or recommendations discussed are solely those of the author(s) and are not endorsed by BMJ. BMJ disclaims all liability and responsibility arising from any reliance placed on the content. Where the content includes any translated material, BMJ does not warrant the accuracy and reliability of the translations (including but not limited to local regulations, clinical guidelines, terminology, drug names and drug dosages), and is not responsible for any error and/or omissions arising from translation and adaptation or otherwise.

Open access This is an open access article distributed in accordance with the Creative Commons Attribution Non Commercial (CC BY-NC 4.0) license, which permits others to distribute, remix, adapt, build upon this work non-commercially, and license their derivative works on different terms, provided the original work is properly cited, appropriate credit is given, any changes made indicated, and the use is non-commercial. See http://creativecommons.org/licenses/by-nc/4.0/.

\section{ORCID iDs}

Razvan Cristescu http://orcid.org/0000-0002-9978-0339

Alexandra Snyder http://orcid.org/0000-0002-2606-3523

\section{REFERENCES}

1 Schumacher TN, Schreiber RD. Neoantigens in cancer immunotherapy. Science 2015;348:69-74.

2 Yarchoan M, Hopkins A, Jaffee EM. Tumor mutational burden and response rate to PD-1 inhibition. N Engl J Med 2017;377:2500-1.

$3 \mathrm{Kim}$ JY, Kronbichler A, Eisenhut M, et al. Tumor mutational burden and efficacy of immune checkpoint inhibitors: a systematic review and meta-analysis. Cancers 2019;11:1798.

4 Osipov A, Lim SJ, Popovic A, et al. Tumor mutational burden, toxicity, and response of immune checkpoint inhibitors targeting PD(L)1, CTLA-4, and combination: a meta-regression analysis. Clin Cancer Res 2020;26:4842-51.

5 Marabelle A, Fakih M, Lopez J, et al. Association of tumour mutational burden with outcomes in patients with advanced solid tumours treated with pembrolizumab: prospective biomarker analysis of the multicohort, open-label, phase 2 KEYNOTE-158 study. Lancet Oncol 2020;21:1353-65.

6 Ribas A, Hamid O, Daud A, et al. Association of pembrolizumab with tumor response and survival among patients with advanced melanoma. JAMA 2016;315:1600-9.

7 Garon EB, Rizvi NA, Hui R, et al. Pembrolizumab for the treatment of non-small-cell lung cancer. N Engl J Med 2015;372:2018-28.

8 Ribas A, Puzanov I, Dummer R, et al. Pembrolizumab versus investigator-choice chemotherapy for ipilimumab-refractory melanoma (KEYNOTE-002): a randomised, controlled, phase 2 trial. Lancet Oncol 2015;16:908-18.

9 Herbst RS, Baas P, Kim D-W, et al. Pembrolizumab versus docetaxel for previously treated, PD-L1-positive, advanced non-small-cell lung cancer (KEYNOTE-010): a randomised controlled trial. Lancet 2016;387:1540-50.

10 Nanda R, Chow LQM, Dees EC, et al. Pembrolizumab in patients with advanced triple-negative breast cancer: phase lb KEYNOTE-012 study. J Clin Oncol 2016;34:2460-7.

11 Muro K, Chung HC, Shankaran V, et al. Pembrolizumab for patients with PD-L1-positive advanced gastric cancer (KEYNOTE-012): a multicentre, open-label, phase $1 \mathrm{~b}$ trial. Lancet Oncol 2016;17:717-26.

12 Plimack ER, Bellmunt J, Gupta S, et al. Safety and activity of pembrolizumab in patients with locally advanced or metastatic urothelial cancer (KEYNOTE-012): a non-randomised, open-label, phase 1b study. Lancet Oncol 2017;18:212-20.

13 Mehra R, Seiwert TY, Gupta S, et al. Efficacy and safety of pembrolizumab in recurrent/metastatic head and neck squamous cell carcinoma: pooled analyses after long-term follow-up in KEYNOTE-012. Br J Cancer 2018;119:153-9.

14 Ott PA, Bang Y-J, Piha-Paul SA, et al. T-cell-inflamed geneexpression profile, programmed death ligand 1 expression, and tumor mutational burden predict efficacy in patients treated with pembrolizumab across 20 cancers: KEYNOTE-028. J Clin Oncol 2019;37:318-27.

15 Bellmunt J, de Wit R, Vaughn DJ, et al. Pembrolizumab as secondline therapy for advanced urothelial carcinoma. $N$ Engl J Med 2017;376:1015-26

16 Bauml J, Seiwert TY, Pfister DG, et al. Pembrolizumab for platinumand cetuximab-refractory head and neck cancer: results from a single-arm, phase II study. J Clin Oncol 2017;35:1542-9.

17 Fuchs CS, Doi T, Jang RW, et al. Safety and efficacy of pembrolizumab monotherapy in patients with previously treated advanced gastric and gastroesophageal junction cancer: phase 2 clinical KEYNOTE-059 trial. JAMA Oncol 2018;4:e180013.

18 Shitara K, Özgüroğlu M, Bang Y-J, et al. Pembrolizumab versus paclitaxel for previously treated, advanced gastric or gastrooesophageal junction cancer (KEYNOTE-061): a randomised, openlabel, controlled, phase 3 trial. Lancet 2018;392:123-33. 
19 Adams S, Schmid P, Rugo HS, et al. Pembrolizumab monotherapy for previously treated metastatic triple-negative breast cancer: cohort A of the phase II KEYNOTE-086 study. Ann Oncol 2019;30:397-404.

20 Matulonis UA, Shapira-Frommer R, Santin AD, et al. Antitumor activity and safety of pembrolizumab in patients with advanced recurrent ovarian cancer: results from the phase II KEYNOTE-100 study. Ann Oncol 2019;30:1080-7.

21 Antonarakis ES, Piulats JM, Gross-Goupil M, et al. Pembrolizumab for treatment-refractory metastatic castration-resistant prostate cancer: multicohort, open-label phase II KEYNOTE-199 study. J Clin Oncol 2020;38:395-405.

22 Cristescu R, Mogg R, Ayers M, et al. Pan-tumor genomic biomarkers for PD-1 checkpoint blockade-based immunotherapy. Science 2018;362 doi:10.1126/science.aar3593

23 Ayers M, Lunceford J, Nebozhyn M, et al. IFN- $\gamma$-related mRNA profile predicts clinical response to PD-1 blockade. J Clin Invest 2017;127:2930-40.

24 Panda A, Betigeri A, Subramanian K, et al. Identifying a clinically applicable mutational burden threshold as a potential biomarker of response to immune checkpoint therapy in solid tumors. JCO Precis Oncol 2017;2017:1-13. doi:10.1200/PO.17.00146

25 Friends of Cancer Research TMB Harmonization Working Group. Tissue agnostic TMB clinical cut-off harmonization initiative. Washington, DC, 2020. https://www.focr.org/sites/default/files/ Tissue-Agnostic-TMB_Summary.pdf

26 Samstein RM, Lee C- $\bar{H}$, Shoushtari AN, et al. Tumor mutational load predicts survival after immunotherapy across multiple cancer types. Nat Genet 2019;51:202-6.

27 Alexandrov LB, Nik-Zainal S, Wedge DC, et al. Signatures of mutational processes in human cancer. Nature 2013;500:415-21.

28 Castle JC, Uduman M, Pabla S, et al. Mutation-derived neoantigens for cancer immunotherapy. Front Immunol 2019;10:10.

29 Rizvi NA, Hellmann MD, Snyder A, et al. Cancer immunology. Mutational landscape determines sensitivity to PD-1 blockade in non-small cell lung cancer. Science 2015;348:124-8.

30 Chen Y, Liu Q, Chen Z, et al. PD-L1 expression and tumor mutational burden status for prediction of response to chemotherapy and targeted therapy in non-small cell lung cancer. J Exp Clin Cancer Res 2019;38:193.
$31 \mathrm{Yu} \mathrm{H}$, Chen Z, Ballman KV, et al. Correlation of PD-L1 expression with tumor mutation burden and gene signatures for prognosis in earlystage squamous cell lung carcinoma. J Thorac Oncol 2019;14:25-36.

32 Zhou KI, Peterson B, Serritella A, et al. Spatial and temporal heterogeneity of PD-L1 expression and tumor mutational burden in gastroesophageal adenocarcinoma at baseline diagnosis and after chemotherapy. Clin Cancer Res 2020;26:6453-63.

33 Özdoğan M, Papadopoulou E, Tsoulos N, et al. Comprehensive tumor molecular profile analysis in clinical practice. BMC Med Genomics 2021;14:105.

34 Hellmann MD, Ciuleanu T-E, Pluzanski A, et al. Nivolumab plus ipilimumab in lung cancer with a high tumor mutational burden. $N$ Engl J Med 2018;378:2093-104.

35 Chalmers ZR, Connelly CF, Fabrizio D, et al. Analysis of 100,000 human cancer genomes reveals the landscape of tumor mutational burden. Genome Med 2017;9:34.

36 Feliubadaló L, Tonda R, Gausachs M, et al. Benchmarking of whole exome sequencing and AD hoc designed panels for genetic testing of hereditary cancer. Sci Rep 2017;7:37984.

37 Goodman AM, Kato S, Bazhenova L, et al. Tumor mutational burden as an independent predictor of response to immunotherapy in diverse cancers. Mol Cancer Ther 2017;16:2598-608.

38 Georgiadis A, Durham JN, Keefer LA, et al. Noninvasive detection of microsatellite instability and high tumor mutation burden in cancer patients treated with PD-1 blockade. Clin Cancer Res 2019;25:7024-34.

39 Stenzinger A, Allen JD, Maas J, et al. Tumor mutational burden standardization initiatives: recommendations for consistent tumor mutational burden assessment in clinical samples to guide immunotherapy treatment decisions. Genes Chromosomes Cancer 2019;58:578-88.

40 Valero C, Lee M, Hoen D, et al. Response rates to anti-PD-1 immunotherapy in microsatellite-stable solid tumors with 10 or more mutations per Megabase. JAMA Oncol 2021;7:739-43.

41 McGrail DJ, Pilié PG, Rashid NU, et al. High tumor mutation burden fails to predict immune checkpoint blockade response across all cancer types. Ann Oncol 2021;32:661-72.

42 Rousseau B, Foote MB, Maron SB, et al. The spectrum of benefit from checkpoint blockade in hypermutated tumors. $N$ Engl $\mathrm{J}$ Med 2021;384:1168-70. 